\title{
Relación entre innovación y competitividad de los emprendimientos del sector no financiero de la economía popular y solidaria
}

Relationship between Innovation and Competitiveness in the Non-financial Ventures of the Popular and Solidarity Economy

Relação entre inovação e competitividade nos empreendimentos do setor financeiro da Economia Popular e Solidária

\author{
Maribel Maya Carrillo \\ Universidad de las Fuerzas Armadas ESPE. Quito, Ecuador \\ ammaya@espe.edu.ec \\ https://orcid.org/0000-0001-5795-1593 \\ Betsabé Pila Jaramillo \\ Universidad de las Fuerzas Armadas ESPE. Quito, Ecuador \\ bepila@espe.edu.ec \\ https://orcid.org/0000-0002-5246-1154 \\ Valentina Ramos Ramos \\ Escuela Politécnica Nacional. Quito, Ecuador \\ valentina.ramos@epn.edu.ec \\ https://orcid.org/0000-0001-7803-8461
}

DOI: https://doi.org/10.32719/25506641.2022.11.2

Recibido: 15 de enero de 2021 - Revisado: 12 de marzo de 2021

Aceptado: 3 de mayo de 2021 - Publicado: 1 de enero de 2022

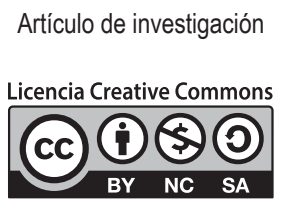




\section{Resumen}

La dinámica empresarial actual exige a los emprendimientos implementar procesos de innovación para generar valor para los clientes, ganar competitividad y ser sostenibles en el mercado. Este trabajo realiza un diagnóstico de las actividades, capacidades y resultados de innovación y la relación con los factores de competitividad de los emprendimientos del sector no financiero popular y solidario que permita comprender la realidad actual y la base para la toma de decisiones futuras incidiendo en el crecimiento empresarial. La investigación es no experimental de enfoque cuantitativo y correlacional para el cual se aplicaron encuestas a los presidentes de 68 emprendimientos del cantón Quito entre marzo y junio de 2020. En los resultados, predominó el nivel medio de innovación y de competitividad; en la capacidad de innovación, se destaca el aprendizaje organizacional. En innovación tecnológica, se evidencia la mejora en la calidad de productos y nuevos métodos productivos; en innovación no tecnológica, se han implementado nuevos canales de comunicación, comercialización y nuevas prácticas empresariales. En competitividad, los emprendimientos están enfocados en recursos humanos y comercialización. Se evidencia una correlación positiva fuerte entre innovación y competitividad.

Palabras clave: Emprendimiento, innovación, competitividad, economía popular y solidaria. JEL: O31 Innovación e invención: procesos e incentivos.

\section{Abstract}

Current business dynamics require ventures to implement innovation processes to generate value for customers, gain competitiveness, and be sustainable in the market. This study analyzes the activities, capacities, and results of innovation and the relationship with the competitiveness factors of ventures in the popular and solidary non-financial sector, in order to understand the current reality and the basis for future decision-making with an influence in business growth. This research is non-experimental, using a quantitative and correlational approach by conducting surveys to the presidents of 68 ventures in the Canton Quito between the months of March and June 2020. The results showed a predominant medium level of innovation and competitiveness, with organizational learning standing out in the capacity for innovation. In terms of technological innovation, improvements in the quality of products and new production methods can be identified; as for non-technological innovation, new communication and marketing channels and new business practices have been implemented. With regard to competitiveness, the ventures are focused on human resources and marketing. There is a strong positive correlation between innovation and competitiveness.

Keywords: Entrepreneurship, innovation, competitiveness, popular and solidarity economy. JEL: O31 Innovation and invention: processes and incentives. 


\section{Resumo}

A dinâmica empresarial atual exige que os empreendimentos implementem processos de inovação para gerar valores para os clientes, ganhar competitividade e ser sustentáveis para o mercado. Este lavor realiza um diagnóstico das atividades, capacidades e resultados de inovação e a relação com os fatores de competitividade dos empreendimentos do setor financeiro popular e solidário que permitem compreender a realidade atual e que são um suporte para a tomada de decisões futuras incidindo no crescimento empresarial. A pesquisa é não experimental, de enfoque quantitativo e concernente, para a qual se aplicaram enquetes aos presidentes de 68 empreendimentos do Canton de Quito entre os meses de março a junho de 2020. Nos resultados, predominou um nível mediano de inovação e de competitividade, na capacidade de inovação destacou-se o aprendizado organizacional. Em inovação tecnológica evidenciaram-se a melhoria da qualidade dos produtos e novos métodos produtivos e em inovação não tecnológica, foram implementados novos canais de comunicação, comercialização e novas práticas empresariais. Em competitividade, os empreendimentos enfocam-se na área de recursos humanos e comercialização. Evidencia-se uma correlação positiva, forte entre inovação e competitividade.

Palavras-chave: Empreendimento, inovação, competitividade, economia popular e solidária. JEL: O31 Inovação e invenção: processos e incentivos.

\section{Introducción}

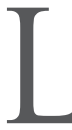

a globalización en las últimas décadas ha significado grandes cambios a nivel social, económico, cultural y ambiental, de manera mundial y en relación directa con el desarrollo de un país (Solleiro y Castañón 2015). Este fenómeno ha obligado a los emprendimientos a ajustarse a un entorno altamente competitivo, donde la innovación se convierte en un factor fundamental para que se establezcan en el mercado o desaparezcan (Schnarch 2007).

Los emprendimientos son proyectos que se desarrollan con diferentes fines, los cuales pueden ser sociales, económicos o políticos y poseen principalmente características de innovación, que permiten satisfacer necesidades de la sociedad de manera regular a cambio de obtener un beneficio económico, contribuyendo al desarrollo local, regional y nacional (Drucker 1985; Duarte y Ruiz 2009). El emprendimiento está relacionado con aquella forma de identificar oportunidades que sean rentables en el mercado (Abd y Samad 2016). 
Según Estrada, García y Sánchez (2009), el éxito de algunos emprendimientos se debe a la importancia que se le ha prestado a la innovación en productos, procesos y la gestión tecnológica para diferenciarse de su competencia. Sin embargo, en América Latina los esfuerzos para implementar innovación aún tienen un progreso lento y claramente muestran una brecha significativa si se los compara con países de otras regiones; esto se debe a diferentes razones, como el limitado acceso al financiamiento que impide en gran medida invertir en temas de innovación (OMPI 2019).

En este contexto, en el Ecuador, la actividad emprendedora temprana (TEA) la cual mide negocios nacientes y nuevos, según el Global Entrepreneurship Monitor (GEM 2019), alcanzó el 36,2\%, una de las tasas más altas de la región; sin embargo, pocos emprendedores ecuatorianos crean nuevos productos y procesos para competir a nivel local e internacional, lo que evidencia un nivel de innovación bajo en comparación a otros países de la región.

En esta realidad ecuatoriana, encajan los emprendimientos del sector de la economía popular y solidaria (EPS), el cual, según el Instituto de Economía Popular y Solidaria IEPS (2018), es importante para la economía ecuatoriana debido a que representa el $25,7 \%$ del PIB y genera el $64 \%$ del empleo a nivel nacional; reconoce el valor del cuidado del medioambiente, el trabajo del ser humano y ha logrado mejorar la calidad de vida de la población menos favorecida distinguiéndose de los otros sectores productivos que no funcionan bajo esta filosofía. La presente investigación se enfoca en el sector no financiero conformado por asociaciones, cooperativas, comunidades y organismos de integración, siendo las primeras la forma de organización más utilizada, concentradas en actividades económicas agropecuarias y textiles (Hernández-Naranjo 2018).

En el estudio "Balance de la Economía Popular y Solidaria 2017 en el Ecuador" (Torres, López y Alemán 2017), se menciona que los principales problemas que atraviesa este sector están relacionados con la inversión, innovación y acceso al mercado, lo cual se desencadena a partir del limitado acceso al crédito debido a los requisitos que exigen las instituciones financieras.

El objetivo principal de esta investigación es evaluar la actividad, capacidad y resultados de innovación de los emprendimientos del sector de la EPS 
en el cantón Quito, y su relación con el nivel de competitividad, mediante una investigación cuantitativa y correlacional.

A continuación, se realiza un abordaje conceptual de la EPS, innovación y competitividad, alineado al objetivo de la presente investigación.

\section{Estado de la cuestión}

\section{Economía popular y solidaria}

La Ley Orgánica de la Economía Popular y Solidaria en su art. 1, define a la EPS como aquella forma de organización económica en la que los miembros, de manera individual o colectivamente, se organizan para realizar actividades productivas, de intercambio, comercialización, financiamiento y consumo de bienes y servicios con el fin de satisfacer necesidades y generar ingresos bajo los principios de solidaridad, cooperación y reciprocidad beneficiando al trabajo y al ser humano como sujeto y fin de su actividad (SEPS 2018).

Desde el enfoque económico, Schumpeter (1934) posicionó a la innovación como un proceso dinámico, alineado a la estrategia empresarial y la cualidad diferenciadora frente a la competencia. De manera complementaria, este autor afirmó que los cambios tecnológicos son la principal causa de los ciclos que vive una economía al adoptar un nuevo conocimiento y su aplicación en la actividad empresarial. En este contexto, se define a la innovación como la acción y efecto que permiten mejoras significativas en las cosas a través de cambios novedosos y constituye la clave del éxito para las economías actuales siendo la tecnología el principal factor (Mendizábal 2002).

En la actualidad, la innovación es considerada como una de las fuentes más importantes de ventaja competitiva, debido a que conlleva a la mejora continua de productos y procesos que permite a las empresas ser más eficientes, tener un crecimiento acelerado y mayor rentabilidad (Helfat y Peteraf 2003; Reichstein y Salter 2006; Artz et al. 2010). Las empresas, para reforzar la ventaja competitiva, tienen que potenciar su capacidad de innovación mediante el desarrollo y comercialización de nuevas tecnologías de manera 
efectiva, a través de innovaciones exitosas en el mercado (Börjesson, Elmquist y Hooge 2014).

La actividad innovadora está implícita en todo el proceso de innovación con el fin de que las empresas alcancen un mejor nivel de competitividad a través de la reducción de sus costos, mejoras en la calidad y creación de nuevos productos, por lo cual incurren en esfuerzos innovativos (EC INEC 2015). Estas actividades no solo hacen referencia a los esfuerzos que realizan las empresas para la generación, adquisición o adaptación de conocimiento, sino a su vez invierte en otros esfuerzos, como tecnologías o mejoras en el recurso humano, todas aquellas acciones que permiten crecer en innovación (Lugones 2016).

Los esfuerzos de innovación son puestos en marcha debido a la capacidad de innovación que tienen las empresas, vista esta como la principal fuente para incrementar la productividad, competitividad y crecimiento empresarial (Sánchez, García y Mendoza 2015), debido a que son aquellas habilidades que desarrolla una empresa para producir innovación a través del aprendizaje permanente, la transformación del conocimiento, motivando a la creatividad activa de sus trabajadores y la disponibilidad de recursos internos como externos al alcance de la empresa y de esta manera identificar oportunidades en el mercado (Iddris 2016; Yam Lo, Tang y Lau 2004).

Las capacidades de innovación están fuertemente relacionadas con los tipos de innovación, especialmente de productos y procesos, ya que permite reducir tiempo y mejorar la eficiencia de los productos (Arias, Durango y Millán 2015). Esta tipología se evidencia en los resultados de innovación que se componen de innovación tecnológica (productos y procesos), gestiona en los sistemas técnicos de una organización y se encuentra relacionada con las actividades básicas o primarias en todo el proceso de creación de valor (Damanpour y Evan 1984) y de innovación no tecnológica (marketing y organizacional); estos tipos de innovación logran cambios significativos o totalmente nuevos en prácticas empresariales, mejoras en los puestos de trabajo, relaciones con clientes o proveedores, diseños y empaques diferentes (OCDE y Eurostat 2005).

Los emprendimientos que aplican innovación logran obtener ventajas competitivas sostenibles, entre estas se puede mencionar la calidad de productos, flexibilidad para responder oportunamente a los cambios en el 
mercado, reducción de costos y tiempo en el proceso productivo, atributos importantes al momento de competir (Cervilla de Oliviere 2005).

Porter (1990) explica que la competitividad es aquella capacidad que permite sostener e incrementar la participación en los mercados y a su vez incidir en el nivel y calidad de vida de la población; esto solo se logra cuando las empresas son más productivas y contribuyen al desarrollo de un país.

En la presente investigación se consideró la perspectiva de competitividad a nivel empresarial, la que se refiere a que una empresa es competitiva cuando se plantea mejorar su calidad, productividad, obtener mayor grado de eficiencia, captar mayor cuota de mercado y obtener beneficios económicos sobre la inversión que han realizado; es decir, las empresas son competitivas cuando poseen factores internos o ventajas diferenciadoras frente a otras empresas que tienen similares o iguales productos para que puedan crecer y sostenerse en el mercado (Padilla 2006; Solleiro y Castañón 2015; Pech y Morales 2000).

Para determinar la competitividad es necesario considerar elementos que influyen a nivel de la empresa y fuera de esta; en este contexto se consideran factores internos y externos como parte del estudio de competitividad empresarial. En los factores internos se estableció seis dimensiones: i) planificación estratégica; ii) producción y operaciones; iii) aseguramiento de calidad; iv) comercialización; v) contabilidad y finanzas; y vi) recursos humanos (Ibarra, González y Demuner 2017). Mientras que para los factores externos se consideraron dos dimensiones: i) gestión ambiental; y ii) normas y reglamentos técnicos (Saavedra 2017).

Los factores internos analizan dimensiones que se encuentran interconectadas, las cuales permiten realizar un diagnóstico organizacional para identificar fortalezas y debilidades de las áreas o departamentos y tomar decisiones para mejorar el desempeño empresarial (Ibarra, González y Demuner 2017; Bocarando, Mendoza y Castañeda 2016). Por otro lado, las empresas deben considerar factores externos del mercado en que desean competir, ya que de esta manera las empresas logran desarrollar procesos de aprendizaje e incrementar su eficiencia (Esser et al. 1996; Saavedra 2017).

La relación que existe entre innovación y competitividad ha demostrado que la innovación es clave para generar actividades de valor e influir en el crecimiento y competitividad empresarial, debido a que está presente en 
diferentes fases como la invención, innovación, difusión, transferencia tecnológica y a su vez puede llegar a tener grandes efectos socioeconómicos, que permite el desarrollo y transformación de ideas en productos o procesos nuevos o mejorados que la demanda requiera para satisfacer sus necesidades (Abd y Samad 2016). Así también, Distanont y Khongmalai (2018) afirman que la innovación es una herramienta estratégica para mejorar la competencia y éxito en los negocios logrando un desarrollo sostenible.

En la figura 1, se resumen las variables a investigar, con la finalidad de comprobar la hipótesis de si existe o no correlación entre los resultados de innovación y la competitividad en los emprendimientos asociativos no financieros de la EPS.

\section{Material y métodos}

La investigación tiene un enfoque cuantitativo que busca establecer la fuerza de asociación o correlación que tienen las variables de una muestra que permitan explicar mejor las causas de determinado fenómeno (Pita y Pértegas 2002). El diseño de la investigación fue de tipo no experimental, con alcance descriptivo y correlacional de acuerdo con Hernández-Sampieri, Fernández y Baptista (2014) que afirman que este tipo de investigación se basa en la observación de las variables sin manipularlas para analizar su comportamiento y permite el relacionamiento de variables. La hipótesis es: "Existe una relación positiva entre los resultados de innovación y competitividad".

\section{Participantes}

Emprendimientos asociativos del sector no financiero popular y solidario, empleando el catastro obtenido de la SEPS (2019), el cual permitió identificar las actividades económicas con mayor presencia en el cantón Quito: 28 textiles (41\%), 17 agropecuarias (25\%), 15 artesanales (22\%) y 8 alimenticias (12\%), con un total de 68 emprendimientos. Las encuestas se apli- 


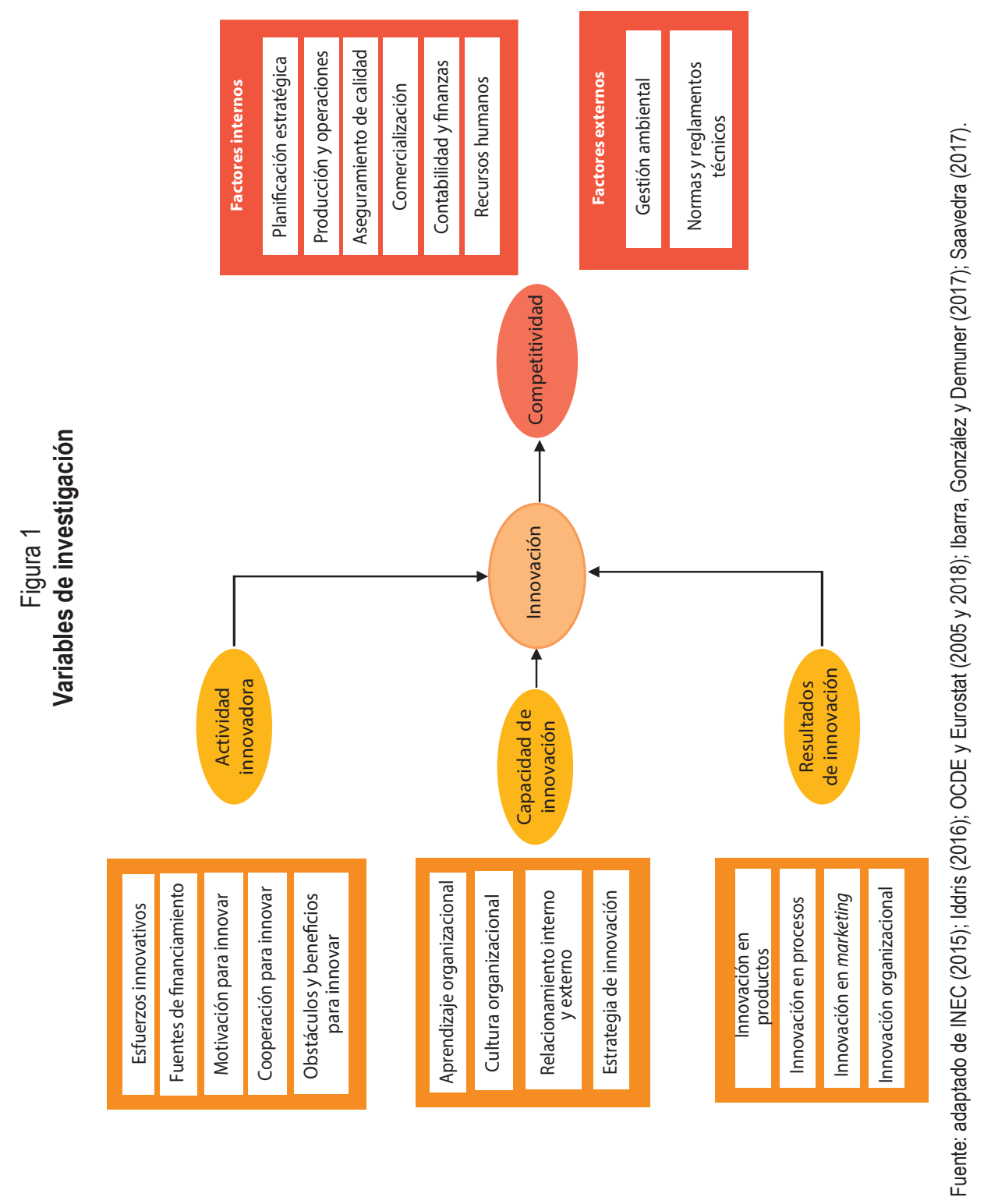


caron a los representantes de cada emprendimiento siendo 41 mujeres (60\%) y 27 hombres $(40 \%)$ que cumplieron con las características de actividades económicas antes mencionadas y forman parte de la EPS.

\section{Instrumentos}

Se emplearon tres instrumentos complementarios para medir la innovación. El primero tomó como base diversas preguntas de la Encuesta Nacional de Actividades de Ciencia, Tecnología e Innovación (ACTI) de 2012-2014, la cual tiene como objetivo medir la actividad innovadora y determinar el nivel de innovación de los emprendimientos. Los componentes que se analizaron son: actividades, fuentes de financiamiento, motivación, factores de cooperación, beneficios y obstáculos para innovar. Se empleó una escala de Likert de 5 puntos para cada pregunta (siendo 1 muy en desacuerdo y 5 muy de acuerdo).

El segundo instrumento se adaptó de la investigación de Iddris (2016) que trata sobre las capacidades de innovación con varias dimensiones y sus respectivos indicadores; en este caso se procedieron a seleccionar y adaptar las más relevantes para el estudio. El objetivo de este instrumento es identificar cuál es la dimensión más empleada por los emprendimientos para evaluar su capacidad de innovación. Los componentes que se analizaron son: aprendizaje organizacional, cultura organizacional, relacionamiento interno y externo, estrategia de innovación. Se empleó una escala de Likert de 5 puntos para cada pregunta (siendo 1 muy en desacuerdo y 5 muy de acuerdo).

Finalmente, para el instrumento de resultados de innovación se utilizaron las definiciones del OCDE y Eurostat 2018 y OCDE y Eurostat 2005 que tienen por objetivo determinar en qué tipo de innovación incursionan los emprendimientos. Este instrumento considera a la innovación en productos, procesos, marketing y organizacional. Se empleó una escala de Likert de 5 puntos para cada pregunta (siendo 1 muy en desacuerdo y 5 muy de acuerdo).

En el caso de la variable de competitividad, se consideró el trabajo realizado por Ibarra, González y Demuner (2017) para analizar factores internos, el cual tiene por objetivo identificar los elementos con mayor importancia para los emprendimientos y el nivel de competitividad del grupo de análisis, mientras que, para los factores externos se adoptó el estudio realizado por 
Santillán (2010) que tiene por objetivo analizar si los elementos del mercado son determinantes para el nivel de competitividad alcanzado. Se empleó una escala de Likert de 5 puntos para cada pregunta (siendo 1 muy en desacuerdo y 5 muy de acuerdo).

\section{Proceso}

\section{a) Validez del contenido}

Conforme al enfoque de Cabero y Llorante (2013), la investigación empleó el juicio de expertos para la validación de instrumentos. El comité lo conformaron, dos gerentes de emprendimientos alimenticios y agropecuarios, dos docentes universitarios y un especialista de emprendimientos e innovación, lo cuales consideraron cinco criterios de evaluación: claridad, coherencia, relevancia, suficiencia y una casilla de sugerencias.

\section{b) Confiabilidad interna de los instrumentos}

El Alfa de Cronbach se empleó para medir la consistencia interna de los ítems de capacidades y resultados de innovación, así como también de competitividad, logrando un resultado en todos los casos mayor a 0,789 , lo que permitió comprobar que los instrumentos son válidos y confiables.

\section{c) Procesamiento y análisis de datos}

La aplicación de los distintos instrumentos a los representantes de los emprendimientos se realizó mediante correo electrónico. El análisis estadístico de datos utilizó el programa SPSS V.22. En la relación entre innovación y competitividad se empleó el coeficiente de correlación de Pearson.

En la interpretación de datos, se consideró cada dimensión al 100\% y se calculó su impacto en cada una de ellas, conforme a los resultados obtenidos por los emprendimientos en cada sector. 


\section{Resultados y discusión}

Los resultados obtenidos de los 68 emprendimientos asociativos de la EPS en los sectores textil, agropecuario y artesanal y alimenticio, se presentan a continuación:

\section{Actividad innovadora}

En la tabla 1 se observa que, al analizar cada una de las dimensiones al $100 \%$ por actividad económica, los esfuerzos innovativos de los emprendimientos alimenticios alcanzan un $45 \%$, seguido por emprendimientos agropecuarios y artesanales con un $31 \%$; se destaca en esta dimensión la capacitación al personal, al desarrollar nuevas habilidades en los trabajadores, así como también, la adquisición de nueva maquinaria y equipos para mejorar la producción.

Los emprendimientos analizados han demostrado su interés por capacitar al personal para fortalecer el aprendizaje tecnológico y desarrollo de nuevas habilidades en los trabajadores, lo que coincide con lo expuesto por Ortega (2019).

En la dimensión de fuentes de financiamiento, los recursos propios se muestran como la principal fuente para innovar en los emprendimientos sin considerar la actividad económica que realicen. En un porcentaje menor, se identifica el uso de financiamiento que proviene de la banca privada o cooperativas.

\section{Tabla 1}

\section{Actividad innovadora por tipo de emprendimiento}

\begin{tabular}{|l|c|c|c|c|}
\hline $\begin{array}{c}\text { Dimensión / } \\
\text { Tipo de emprendimiento }\end{array}$ & $\begin{array}{c}\text { Textil } \\
\%\end{array}$ & $\begin{array}{c}\text { Agropecuario } \\
\%\end{array}$ & $\begin{array}{c}\text { Artesanal } \\
\%\end{array}$ & $\begin{array}{c}\text { Alimenticio } \\
\%\end{array}$ \\
\hline Esfuerzos innovativos & 26 & 31 & 31 & 45 \\
\hline Fuentes de financiamiento & 78 & 80 & 91 & 75 \\
\hline Motivación para innovar & 66 & 65 & 68 & 63 \\
\hline Obstáculos para innovar & 69 & 65 & 66 & 64 \\
\hline Beneficios obtenidos & 67 & 68 & 66 & 64 \\
\hline
\end{tabular}

Elaboración propia. 
En la dimensión de motivación para innovar, se destaca la importancia de aprovechar ideas generadas al interior del emprendimiento para identificar nuevos mercados. Obtuvo en promedio en esta dimensión el 65,5\% en las distintas actividades económicas. En los obstáculos para innovar se observa que más del $60 \%$ de los emprendimientos presentan problemas al momento de implementar innovación, siendo la principal causa la falta de fondos que tiene el emprendimiento; esto guarda una fuerte relación con la dimensión del financiamiento puesto que al solo contar con recursos propios el nivel de innovación se limita; del mismo modo, el mercado está dominado por empresas establecidas con gran posicionamiento.

En la dimensión de beneficios obtenidos al innovar, los emprendimientos han logrado aumentar la calidad y variedad de los productos que ofrecen en el mercado, destacándose en esta dimensión los emprendimientos del sector agropecuario.

Finalmente, en la dimensión de cooperación para innovar, se analizan los tipos de ayuda que han realizado los diferentes actores: clientes y consumidores, universidades y proveedores con los emprendimientos. E1 41\% de emprendimientos textiles recibieron apoyo, destacándose la investigación y desarrollo e información proveniente de clientes y consumidores. De este modo, se puede concluir que el $59 \%$ restante de los actores u organismos no colaboraron con este tipo de emprendimiento.

Tabla 2

Actividad innovadora: dimensión cooperación para innovar por tipo de emprendimiento

\begin{tabular}{|l|r|c|c|c|}
\hline \multicolumn{1}{|c|}{ Tipo de ayuda } & $\begin{array}{c}\text { Textil } \\
\%\end{array}$ & $\begin{array}{c}\text { Agropecuario } \\
\%\end{array}$ & $\begin{array}{c}\text { Artesanal } \\
\%\end{array}$ & $\begin{array}{c}\text { Alimenticio } \\
\%\end{array}$ \\
\hline Asistencia técnica & 6 & 7 & 6 & 11 \\
\hline Información & 10 & 9 & 9 & 7 \\
\hline Ingeniería y diseño & 7 & 6 & 7 & 11 \\
\hline Investigación y desarrollo & 10 & 10 & 17 & 13 \\
\hline Prueba de productos & 8 & 10 & 4 & 8 \\
\hline Total ayuda percibida & 41 & 42 & 43 & 50 \\
\hline
\end{tabular}

Elaboración propia. 
En los emprendimientos agropecuarios, se observa que un $42 \%$ de los actores (universidades y clientes o consumidores), colaboraron en investigación y desarrollo y prueba de productos. Mientras que un $43 \%$ apoyan a emprendimientos artesanales en investigación y desarrollo con un $17 \%$, según la información obtenida por parte de los proveedores.

Por último, en los emprendimientos alimenticios se puede observar que los clientes y consumidores, como actores de cooperación, han ayudado a desarrollar acciones que fomenten la investigación y desarrollo en un $13 \%$.

La cooperación entre actores permite articular conocimiento que potencia actividades de innovación en los emprendimientos, sin embargo, esta articulación depende de la apertura de los empresarios a colaborar y de políticas que faciliten ese apoyo de beneficio mutuo.

\section{Nivel de innovación}

Sobre los resultados obtenidos de las dimensiones de la actividad innovadora en los emprendimientos analizados, se asignaron pesos a las respuestas obtenidas entre 1 y 5 puntos, tomando como referencia el trabajo realizado por Romero, Rébori y Camino (2010). De esta forma, se obtuvo un total de 209 puntos máximos por cada emprendimiento, lo que permitió categorizarlos en tres niveles: nivel bajo, de 0 a 68 puntos, un total de 9 emprendimientos; nivel medio, de 69 a 118 puntos, 58 emprendimientos, y en el nivel alto no existió ningún emprendimiento. Uno de los factores que pueden influir en estos resultados es la escasa cooperación entre organismos de investigación, ciencia y tecnología, laboratorios de investigación y desarrollo y universidades.

La mayor parte de emprendimientos analizados han demostrado ser medianamente innovadores, como la mayoría de las mipymes, hacen esfuerzos para mejorar la calidad y aumentar la variedad de los productos que se comercializan mediante la adquisición de maquinaria y equipo, y que estos cambios permitan modernizar el proceso productivo e incrementar el desarrollo tecnológico interno (Ortega 2019). Sin embargo, los emprendimientos en Ecuador no logran generar procesos y productos altamente novedosos; es 
evidente la falta de diferenciación y creación de valor en el mercado nacional e internacional.

Otro factor que puede ser influyente en el nivel de innovación alcanzado por los emprendimientos, es el financiamiento; los limitados recursos propios para adquirir más y mejor tecnología con el fin de incrementar su producción y responder rápidamente a las necesidades del mercado es insuficiente y dificulta la recuperación de la inversión. Asimismo, el complejo acceso a financiamiento externo de instituciones públicas o privadas, se ha convertido en un importante motivo para cerrar las puertas de negocios ecuatorianos (GEM 2020). Debido a esta realidad, es necesario que el Estado apoye a este sector prioritario mediante alternativas de financiamiento como capital semilla, capital riesgo e inversión ángel como lo señala la Ley Orgánica de Emprendimientos e Innovación (EC 2020, art. 2).

La cooperación deficiente con otros actores del ecosistema de emprendimiento e innovación, dificulta dinamizar los emprendimientos de la EPS. Uno de los elementos que influyen significativamente para generar innovaciones son los resultados de procesos de investigación y desarrollo, debido a que se afirma que las empresas que colaboran en $\mathrm{I}+\mathrm{D}$ reducen costos y sus resultados se emplean en la estrategia empresarial para hacerla más competitiva (López, Martínez y Céspedes 2013).

La colaboración entre la empresa y la universidad, como generadora de conocimiento, permite a los emprendedores acceder a infraestructura tecnológica de forma más económica, con menos riesgo y mayor agilidad, tal y como se demuestra en un estudio realizado en Reino Unido en empresas químicas y de electricidad las cuales han logrado tener un alto nivel de intensidad competitiva e investigación, mediante el empleo de todo tipo de información de fuentes externas. En este caso, las universidades tienen la capacidad de colaborar mediante planes de negocios, prueba de productos e impulsar la investigación y desarrollo dentro de las empresas para mejorar la ventaja competitiva (Vélez, Afcha y Bustamante 2019).

Finalmente, la colaboración vertical, es decir, la colaboración entre clientes y proveedores, es una fuente muy empleada por los emprendedores y empresas pequeñas, ya que permite reducir el tiempo de producción y entrega, estar a la vanguardia de nueva tecnología en el mercado, mejorar la calidad 
de productos, identificar la forma correcta para comercializar e introducir nuevas innovaciones en los productos (Balarezo y Ortega 2018).

Los emprendimientos de la EPS, para mejorar su nivel de innovación, requieren impulsar sus esfuerzos innovativos, crear redes de colaboración con actores generadores de conocimiento, así como promover políticas que incentiven el financiamiento público y privado, para de esta manera crear y mejorar productos y procesos más competitivos generadores de cambio.

\section{Capacidad de innovación}

En el análisis de las capacidades de innovación, como se evidencia en la tabla 3, la dimensión de aprendizaje organizacional es la que mejor se gestiona en los emprendimientos de tipo textil, agropecuario y alimenticio, donde se destaca el reconocimiento de experiencias y lecciones aprendidas para prevenir problemas futuros, es decir, el conocimiento que se puede adquirir en la práctica para aplicarlo en nuevos acciones y proyectos. La dimensión de cultura organizacional muestra que los emprendimientos de tipo textil se preocupan en mayor medida por desarrollar proyectos o planes para que los trabajadores propongan ideas creativas e innovadoras que permita incrementar el nivel de innovación; así también han logrado desarrollar un clima laboral en el cual los trabajadores se sientan comprometidos con la organización, compartiendo información entre niveles operativos y de gerencia con el fin de mantener una buena comunicación y generación de ideas innovadoras que puedan ser aplicables de manera exitosa.

\section{Tabla 3}

Análisis de la capacidad de innovación por dimensión y emprendimiento

\begin{tabular}{|l|c|c|c|c|}
\hline \multicolumn{1}{|c|}{ Dimensión } & $\begin{array}{c}\text { Textil } \\
\%\end{array}$ & $\begin{array}{c}\text { Agropecuario } \\
\%\end{array}$ & $\begin{array}{c}\text { Artesanal } \\
\%\end{array}$ & $\begin{array}{c}\text { Alimenticio } \\
\%\end{array}$ \\
\hline Aprendizaje organizacional & 75 & 73 & 68 & 72 \\
\hline Cultura organizacional & 71 & 64 & 66 & 64 \\
\hline Relacionamiento interno y externo & 74 & 67 & 70 & 61 \\
\hline Estrategia de innovación & 74 & 70 & 64 & 63 \\
\hline
\end{tabular}

Elaboración propia. 
La dimensión de relacionamiento interno y externo obtiene mejores resultados en los emprendimientos textiles $(74 \%)$ y artesanales $(70 \%)$, esto se debe a que se ha fortalecido la comunicación entre la empresa, cliente, proveedores y los mismos trabajadores, lo que ha permitido realizar cambios significativos en la cadena de suministro y fidelizar al cliente.

Finalmente, en la estrategia de innovación se destacan los emprendimientos de tipo textil y agropecuario, son los que muestran un mejor resultado al momento de implementar acciones que permiten desarrollar innovación a nivel interno, mediante propuestas de incidencia pública y privada, y recursos para financiar acciones en su planificación estratégica enfocadas a incrementar el nivel de innovación.

La capacidad de innovación es la principal fuente para incrementar la productividad, competitividad y crecimiento a nivel empresarial (Sánchez, García y Mendoza 2015). Lograr la integración de todas las dimensiones para potenciar la capacidad de innovación es un reto al que se enfrentan todas las mipymes para obtener mejores indicadores de innovación y competitividad.

En América Latina, un estudio realizado por la CEPAL (2007) indica que no existe un aporte significativo para desarrollar y fortalecer capacidades de innovación en las empresas, debido a la falta de integración de los actores del ecosistema y el desinterés de los empresarios por dinamizar sus organizaciones. En Ecuador, según el GEM (2020), la capacidad de innovación es uno de los temas más desafiantes para los empresarios debido al demostrado retroceso para construir empresas innovadoras y con un alto crecimiento tecnológico en los últimos años. Por lo tanto, es necesario potenciar los dominios que integran las capacidades de innovación en las nuevas y actuales empresas.

\section{Resultados de innovación}

En términos generales la innovación es una herramienta estratégica para mejorar la competencia y éxito en los negocios logrando un desarrollo sostenible (Distanont y Khongmalai 2018). En la presente investigación, los resultados de innovación tecnológica y no tecnológica se los analiza en función a los últimos cinco años por sector de emprendimiento. En la tabla 4 se evidencia la información de los elementos de innovación tecnológica (productos y procesos). 
Tabla 4

Innovación tecnológica por emprendimiento

\begin{tabular}{|l|c|c|c|c|}
\hline \multicolumn{1}{|c|}{ Dimensiones } & $\begin{array}{c}\text { Textil } \\
\%\end{array}$ & $\begin{array}{c}\text { Agropecuario } \\
\%\end{array}$ & $\begin{array}{c}\text { Artesanal } \\
\%\end{array}$ & $\begin{array}{c}\text { Alimenticio } \\
\%\end{array}$ \\
\hline Innovación en productos & 62 & 66 & 65 & 73 \\
\hline Innovación en procesos & 64 & 66 & 60 & 64 \\
\hline
\end{tabular}

Elaboración propia.

La innovación en productos que se observa en la tabla 4, identifica que los emprendimientos de tipo alimenticio (70\%) han implementado este tipo de innovación, seguido por emprendimientos agropecuarios con $66 \%$, esto se debe a la mejora en la calidad de los productos, es decir, plantear innovaciones incrementales. Estos resultados se alinean a la investigación, la cual afirma que la innovación incide en el crecimiento y desarrollo de las empresas del sector de alimentos y bebidas del cantón Quito, las cuales han realizado más innovaciones incrementales que radicales o disruptivas (Cadena, Pereira y Pérez 2017).

En el caso de la dimensión de innovación en procesos, se evidencia que los emprendimientos agropecuarios $(66 \%)$, textiles $(64 \%)$ y alimenticios $(64 \%)$ han incorporado nuevos métodos que mejoran la productividad y eficiencia del emprendimiento, como el principal esfuerzo realizado en este tipo de innovación.

La OMPI (2020) señala que la innovación tecnológica ha demostrado ser una de las mayores fuentes de crecimiento y competitividad. En Ecuador, 3 de 4 emprendedores ofrecen productos que no se identifican por los clientes como nuevos, solamente 1 de cada 5 emprendedores han logrado introducir productos nuevos en el mercado nacional pero que ya existían en otros mercados, esto ocurre de la misma forma en la innovación en procesos (GEM 2020).

Por otra parte, los resultados sobre innovación no tecnológica (marketing y organizacional), como se observa en la tabla 5, reflejan que el $71 \%$ de emprendimientos agropecuarios y el $68 \%$ de artesanales han logrado introducir mejoras significativas en innovación en marketing debido a que han identificado nuevos canales de comunicación que permiten la difusión eficiente de 
su catálogo de productos. En un estudio sobre el caso del sector de seguros, considera que la innovación en marketing y la creatividad son aspectos fundamentales para atraer nuevos clientes mediante canales de venta digitales y reinventar su marca para que pueda ser reconocido en un mercado diferente y con ello crear ventaja competitiva (Chen 2006).

Por otro lado, se ha logrado incorporar nuevas prácticas empresariales en emprendimientos agropecuarios con un $64 \%$ y artesanales con un $62 \%$ mediante la introducción de innovación organizacional; esto ha permitido transformar las rutinas diarias de los trabajadores mediante nuevos sistemas de operación y creación de programas empresariales para el desarrollo de los trabajadores. Las innovaciones organizacionales son fundamentales para mejorar la eficiencia y productividad de los trabajadores, adquirir e identificar nuevas fuentes que permitan el intercambio y transformación del conocimiento y optimizar el aprendizaje (Lam 2005).

Tabla 5

Innovación no tecnológica por emprendimiento

\begin{tabular}{|l|c|c|c|c|}
\hline \multicolumn{1}{|c|}{ Dimensiones } & $\begin{array}{c}\text { Textil } \\
\%\end{array}$ & $\begin{array}{c}\text { Agropecuario } \\
\%\end{array}$ & $\begin{array}{c}\text { Artesanal } \\
\%\end{array}$ & $\begin{array}{c}\text { Alimenticio } \\
\%\end{array}$ \\
\hline Innovación en marketing & 66 & 71 & 68 & 64 \\
\hline Innovación organizacional & 61 & 64 & 62 & 58 \\
\hline
\end{tabular}

Elaboración propia.

Debido a la pandemia provocada por la COVID-19, el 61,1\% de los emprendedores ecuatorianos han optado por implementar mayores esfuerzos en cuanto a innovación en marketing mediante el empleo de ventas en línea y ofrecer nuevos productos (GEM 2020). Estas prácticas son evidencia de que los emprendimientos en tiempos de adversidad requieren adaptarse al entorno e innovar en distintas áreas para continuar en el mercado. 


\section{Competitividad empresarial}

Los resultados de competitividad empresarial se presenta en la tabla 6 . En la dimensión de planificación estratégica se observa que más del 50\% de los emprendimientos analizados han mejorado esta dimensión, siendo los de tipo artesanal quienes lideran con un $75 \%$ ya que han definido de manera explícita y clara la misión, visión, valores y objetivos empresariales. Así también, la estrategia más empleada para generar ventaja competitiva es la de diferenciación, los textiles y alimenticios obtuvieron los más altos porcentajes, 50 y $75 \%$ respectivamente.

\section{Tabla 6}

Resultados de competitividad empresarial (factores internos y externos)

\begin{tabular}{|c|c|c|c|c|}
\hline Dimensión & $\begin{array}{c}\text { Textil } \\
\%\end{array}$ & $\begin{array}{c}\text { Agropecuario } \\
\%\end{array}$ & $\begin{array}{c}\text { Artesanal } \\
\%\end{array}$ & $\begin{array}{c}\text { Alimenticio } \\
\%\end{array}$ \\
\hline \multicolumn{5}{|c|}{ Factores internos } \\
\hline Planificación estratégica & 71 & 71 & 75 & 64 \\
\hline Producción y operaciones & 70 & 68 & 71 & 56 \\
\hline Aseguramiento de calidad & 66 & 66 & 61 & 66 \\
\hline Comercialización & 70 & 78 & 76 & 73 \\
\hline Contabilidad y finanzas & 52 & 65 & 60 & 69 \\
\hline Recursos humanos & 69 & 71 & 67 & 68 \\
\hline \multicolumn{5}{|c|}{ Factores externos } \\
\hline Gestión ambiental & 66 & 71 & 71 & 65 \\
\hline Normas y reglamentos técnicos & 73 & 78 & 72 & 73 \\
\hline
\end{tabular}

Elaboración propia.

En la dimensión de producción y operaciones se puede observar que el $70 \%$ de los 28 emprendimientos textiles, y el $71 \%$ de los 15 artesanales, han logrado adquirir materias primas e insumos oportunamente y mantener un control de inventarios. En cuanto a la dimensión de aseguramiento de calidad, más del $60 \%$ de los emprendimientos analizados han logrado 
implementar un grupo de trabajo que se dedique a la mejora continua de los procesos en la empresa.

La dimensión de comercialización en los emprendimientos agropecuarios, con un $78 \%$, y artesanales con $76 \%$, cuentan con políticas de venta definidas y mantienen buenas relaciones comerciales con clientes y proveedores. Asimismo, en la dimensión de contabilidad y finanzas se observa que los emprendimientos agropecuarios, en un $65 \%$, han mejorado, esto se explica al contar con una estructura de costos bien definida; un $69 \%$ de emprendimientos alimenticios, han generado al menos el $25 \%$ de utilidades en el último año.

En relación con los recursos humanos, los emprendimientos agropecuarios, en un $71 \%$ de los 15 analizados, han logrado establecer políticas de seguridad ocupacional con el fin de prevenir riesgos laborales y garantizar de esta forma la salud y bienestar de los trabajadores.

Dentro de los factores externos, en la dimensión de gestión ambiental los emprendimientos de tipo agropecuario y artesanal han gestionado de mejor forma, al ser responsables del impacto ambiental de sus prácticas laborales. Finalmente, en la dimensión de normas y reglamentos técnicos, se evidenció que los emprendimientos, sin diferencia del tipo de actividad económica al que pertenezcan, afirman cumplir con la mayor parte de requisitos necesarios para comercializar sus productos, siendo los de tipo artesanal, con un $78 \%$, los que en mayor medida cumplen las normas.

\section{Nivel de competitividad}

El nivel de competitividad, se determinó a través de la puntuación máxima de cada uno de los emprendimientos en las diferentes dimensiones que ayudan a medir el nivel de competitividad empresarial, tomando como referencia el estudio realizado por Bocarando, Mendoza y Castañeda (2016). De esta forma, se obtuvo un total de 90 puntos máximos por cada emprendimiento, categorizados en tres niveles: bajo, medio y alto. En el nivel bajo se identificaron 9 emprendimientos con un puntaje de 0 a 36 puntos; en el medio, 38 emprendimientos con un puntaje de 37 a 71 puntos, y finalmente, en el nivel alto, 21 emprendimientos con un puntaje entre 71 a 90 puntos. 
Los emprendimientos con un nivel alto de competitividad reflejan mejoras significativas en áreas como planificación estratégica, producción, operaciones y aseguramiento de la calidad. Mientras que, el nivel medio de competitividad se ve explicado por factores más destacados como la comercialización y los recursos humanos, los cuales, según Aragón y Rubio (2005), deben mantenerse o desarrollarse para crear ventaja competitiva. La comercialización es vista como un factor que permite realizar conexiones fuertes entre clientes y empresas, y los recursos humanos permiten definir procesos de reclutamiento eficientes, identificar a los mejores postulantes y adecuar el clima laboral que influya en la productividad (Ibarra, González y Demuner 2017).

Sin embargo, los emprendimientos que se encuentran en el nivel bajo de competitividad muestran un mayor problema en la dimensión de contabilidad y finanzas, lo que indica que los emprendimientos no han logrado establecer sistemas integrales para determinar costos, o una planificación financiera que les permita tomar decisiones a largo, mediano o corto plazo, a esto se le suma un bajo interés para mejorar en cuanto a factores de gestión ambiental, normas y reglamentos técnicos (Ibarra, González y Demuner 2017).

\section{Relación entre resultados de innovación y competitividad}

La relación entre los resultados de innovación y competitividad se evidencia en la tabla 7.

Tabla 7

Correlación entre competitividad y resultados de innovación en un nivel medio de innovación

\begin{tabular}{|l|l|l|}
\hline \multicolumn{3}{|c|}{ Competitividad } \\
\hline Innovación tecnológica & Correlación de Pearson & $0,665^{* *}$ \\
\hline & Sig. (bilateral) & 0 \\
\hline Innovación no tecnológica & Correlación de Pearson & $0,584^{* *}$ \\
\hline & Sig. (bilateral) & 0 \\
\hline
\end{tabular}

Elaboración propia. 
Estos resultados reflejan que la relación de los emprendimientos medianamente innovadores tiene $p \_$valor $>$de 0,05 , tanto para la innovación tecnológica como no tecnológica, por lo cual se acepta la hipótesis, evidenciando que existe una asociación positiva entre los resultados de innovación y competitividad, y que la innovación tecnológica tiene más fuerte correlación. Los resultados de la investigación se alinean al estudio de Zayas, Parra, López y Torres (2015) quienes afirman que la innovación, el desarrollo tecnológico y la capacitación son elementos claves para que la empresa sea competitiva.

En la presente investigación, los resultados de innovación, en el área tecnológica, destacan el interés por mejorar la calidad de productos, así como añadir nuevas características funcionales de acuerdo con las exigencias de los clientes. Se evidencia una correlación fuerte entre innovación tecnológica y competitividad (Zoltán, László y Ainsley 2018).

En innovación no tecnológica se incluyen mejoras a nivel organizacional y estrategias de mercado, se evidencia que la innovación en marketing es la más empleada por los emprendimientos, que ha permitido identificar nuevos canales de comunicación y venta a través de plataformas digitales como tiendas online y redes sociales. Estas acciones son favorables para los emprendimientos del sector de la EPS y muestran un efecto positivo en la competitividad empresarial. En este sentido, Lam (2005) afirma que las innovaciones organizacionales son fundamentales para mejorar la eficiencia y productividad de los trabajadores, adquirir e identificar nuevas fuentes que permitan el intercambio y transformación del conocimiento, y mejorar el aprendizaje y desempeño empresarial (Roberts y Amit 2003).

La investigación comprobó que la innovación tecnológica y no tecnológica influye en la competitividad de los emprendimientos de manera positiva y significante; por tal motivo, el innovar se convierte en una estrategia empresarial importante para lograr ser sostenibles y exitosos en el mercado. 


\section{Conclusiones}

El presente estudio determinó que el $87 \%$ de emprendimientos asociativos de la EPS analizados están en un nivel medio de innovación y el 13\% en nivel bajo, en función a su actividad innovadora. El motivo principal para introducir innovación, indistintamente al sector que pertenezcan, se debe al aprovechamiento de ideas generadas al interior del emprendimiento y a los escasos procesos de I+D y cooperación con otros organismos de apoyo, para el intercambio de conocimiento que les permita generar nuevas estrategias para mejorar significativamente los productos y procesos que ofrecen.

La evaluación de la capacidad de innovación demostró que la dimensión de aprendizaje organizacional de los emprendimientos analizados es la de mayor importancia, puesto que impulsa la generación de nuevos conocimientos, y permite el desarrollo de competencias en los trabadores para evitar futuros errores.

La innovación tecnológica se considera para los emprendimientos un elemento clave para generar actividades de valor e influir en el crecimiento empresarial. En este sentido, se identificó que la mayoría han realizado innovaciones incrementales, mejorando la calidad de productos e introduciendo nuevas características funcionales que permitan responder a las exigencias de los clientes. En los resultados de innovación no tecnológica se determinaron que, debido a la situación que vive el mundo, se han implementado nuevos canales de venta y comunicación a través del uso de redes sociales o creación de tiendas en línea, de esta forma pueden interactuar y conocer mejor a su cliente.

La competitividad de los emprendimientos asociativos de la EPS, tiene un nivel medio en un $56 \%, 31 \%$ alto y $13 \%$ bajo, demostrándose que los factores más relevantes son la comercialización y gestión de recursos humanos. Por un lado, la comercialización ha permitido definir políticas de venta, conexiones fuertes con clientes y proveedores, mientras que, por otro, la gestión de recursos humanos ha priorizado la implementación de políticas de seguridad ocupacional con el fin de evitar accidentes y proteger a los trabajadores en sus labores, además de crear un clima laboral favorable que permita el desarrollo de capacidades y habilidades. 
La innovación tiene un fuerte impacto positivo en la ventaja competitiva, por lo cual las mipymes deben invertir más en innovación a través de la vinculación entre organismos de investigación y universidades que permitan realizar pruebas de productos, desarrollar planes de negocios, asistencia técnica y asesoramiento en temas legales desarrollando capacidades internas para enfrentarse a los desafíos que presenta el mercado.

Este estudio permite dar un vistazo preliminar para analizar la gestión de innovación dentro de los emprendimientos asociativos de la EPS, demostrando que es un elemento clave para el éxito empresarial; cada esfuerzo por innovar es un escalón para mejorar su competitividad y es la manera para lograr mayor sostenibilidad en un mercado dinámico.

Se recomienda, para futuras investigaciones, analizar la política pública y el entorno de los emprendimientos para conocer su influencia en los resultados de innovación y competitividad, y determinar estrategias de mejora para dinamizar este sector.

\section{Referencias}

Abd, Nurul, y Sarminash Samad. 2016. "Innovation and Competitive Advantage: Moderating Effects of Firm Age in Foods Manufacturing SMES in Malaysia". Procedia Economics and Finance 35 (1): 256-266. https://doi.org/10.1016/s2212-5671(16)00032-0.

Aragón, Antonio, y Alicia Rubio. 2005. "Factores explicativos del éxito competitivo: el caso de las pymes del estado de Veracruz". Revista Contaduría y Administración 100 (216): 35-69. https://doi.org/10.22201/fca.24488410e.2005.568.

Arias, José, Carlos Durango y Nora Millán. 2015. "Capacidad de innovación de proceso y desempeño innovador: efecto mediador de la capacidad de innovación de producto". Ad-minister 1 (27): 75-93. https://doi.org/10.17230/ad-minister.27.4.

Artz, Kendall, Patricia Norman, Donald Hatfield y Laura Cardenal. 2010. "Un estudio longitudinal del impacto de la I $+\mathrm{D}$, las patentes y la innovación de productos en el desempeño de las empresas". Journal of Product Innovation Management 27 (5): 725-740. https://doi. org/10.1111/j.1540-5885.2010.00747.x.

Balarezo, Diana, y Mariela Ortega. 2018. "Efecto de la cooperación en actividades de innovación sobre la inversión en innovación y el desempeño innovador de las empresas del Ecuador. Evidencia empírica 2012-2014”. Tesis doctoral, Universidad de Cuenca, Cuenca. https://bit.ly/39RYUBi. 
Bocarando, Juan, Leticia Mendoza y Maribel Castañeda. 2016. "Determinación de un índice de competitividad a nivel micro para el sector comercial, sub sector abarrotes al por menor". Ciencia Administrativa 1 (2): 85-100. https://bit.ly/3h9Pofh.

Börjesson, Sofia, María Elmquist y Sophie Hooge. 2014. "Los desafíos del desarrollo de la capacidad de innovación: aprender de los estudios longitudinales de los esfuerzos de innovación en Renault y Volvo Cars". Journal of Engineering and Technology Management 31 (1): 120-140. https://doi.org/10.1016/j.jengtecman.2013.11.005.

Cabero, Julio, y María Llorante. 2013. "La aplicación del juicio de experto como técnica de evaluación de las tecnologías de la informaciónTIC". Revista de Tecnología de Información y Comunicación de Educación 7 (2): 11-22. https://bit.ly/3plpnw7.

Cadena, Jaime, Nathaly Pereira y Zulay Pérez. 2017. "La innovación y su incidencia en el crecimiento y desarrollo de las empresas del sector alimentos y bebidas del Distrito Metropolitano de Quito". Revista Espacios 40 (22): 17-27. https://bit.ly/3gNQpLY.

Cervilla de Oliviere, María. 2005. "Excelencia operacional mediante la innovación y el mejoramiento continuo de los procesos". Academia Revista Latinoamericana de Administración 61 (1): 34-47. https://bit.ly/38vW6bh.

Chen, Yongmin. 2006. "Marketing Innovation". Journal of Economics \& Management Strategy 15 (1): 101-123. https://doi.org/10.1111/j.1530-9134.2006.00093.x.

Comisión Económica para América Latina y el Caribe (CEPAL). 2007. "Estudio económico de América Latina y el Caribe". Santiago: CEPAL. https://bit.ly/3fQsSc8.

Damanpour, Fariborz, y William Evan. 1984. "Organizational Innovation and Performance: The Problem of Organizational”. Administrative Sciencie Quarrterly 29 (1): 392-409. https://doi.org/10.2307/2393031.

Distanont, Anyanitha, y Orapan Khongmalai. 2018. "The Role of Innovation in Creating a Competitive Advantage". Kasetsart Journal of Social Sciences 30 (1): 1-7. https://doi. org/10.1016/j.kjss.2018.07.009.

Drucker, Peter. 1985. Innovation and Entrepreneurship Practice and Principales.The Practice of Innovation. Nueva York: Harper \& Row. https://bit.ly/2KMuLcQ.

Duarte, Tito, y Myriam Ruiz. 2009. "Emprendimiento una opción para el desarrollo". Scientia Et Technica 15 (43): 326-331. https://bit.ly/37MFtJD.

EC. 2020. Ley Orgánica de Emprendimientos e Innovación. Registro Oficial 151, 28 de febrero. https://bit.ly/3vvJiLU.

Esser, Klaus, Wolfgang Hillebrand, Dirk Messner y Jorg Meyer. 1996. “Competitividad sistémica”. Revista CEPAL 1 (59): 39-52. https://bit.ly/3dNoI2D.

Estrada, Roberto, Domingo García y Víctor Sánchez. 2009. "Factores determinantes del éxito competitivo: estudio empírico en México". Revista Venezolana de Gerencia 14 (46):169182. https://doi.org/10.31876/revista.v14i46.10528.

Global Entrepreneurship Monitor (GEM). 2019. Global Entrepreneurship Monitor. Accedido junio de 2019. https://bit.ly/2JczZOA.

---. 2020. Global Entrepreneurship Monitor. Accedido enero de 2020. https://bit.ly/2RlcQ01. 
Helfat, Constance, y Margaret Peteraf. 2003. "La visión dinámica basada en recursos: ciclos de vida de capacidades". Strategic Management Journal 24 (1): 997-1010. https://doi. org/10.1002/smj.332.

Hernández-Naranjo, Margarita. 2018. "Informe de rendición de cuentas”. Superintendencia de Economía Popular y Solidaria. Accedido julio de 2019. https://bit.ly/3rp34re.

Hernández-Sampieri Roberto, Carlos Fernández y María Baptista. 2014. Metodología de la investigación. Ciudad de México: McGraw-Hill. https://bit.ly/38sQtLh.

Ibarra, Manuel, Lourdes González y María Demuner. 2017. "Competitividad empresarial de las pequeñas y medianas empresas manufactureras Baja California”. Estudios Fronterizos 11 (1): 107-130. https://doi.org/10.21670/ref.2017.35.a06.

Iddris, Faisal. 2016. "Innovation Capability: A Systematic Review and Research Agenda". Interdisciplinary Journal of Information 11 (1): 235-260. https://doi.org/10.28945/3571.

Instituto de Economía Popular y Solidaria (IEPS). 2018. "IEPS y Universidad Politécnica Salesiana organizaron jornada de reflexión sobre la EPS en Quito". Accedido marzo de 2020. https://bit.ly/3dF3ALQ.

Instituto Nacional de Estadística y Censos (INEC). 2015. Actividades de ciencia, tecnología e innovación. Accedido julio 2019. https://bit.ly/3nL5ONp.

Lam, Alice. 2005. The Oxford Handbook of Innovation. Nueva York: Oxford University Press Inc. http://bit.ly/36Fe0sg.

López, Raquel, Javier Martínez y José Céspedes. 2013. “Cooperación y competencia como antecedentes de la innovación de producto. ¿Aplican las empresas nuevas y establecidas una lógica diferencida?". Investigaciones Europeas de Dirección y Economía de la Empresa 19 (1): 53-62. https://doi.org/10.1016/j.iedee.2012.09.001.

Lugones, Gustavo. 2016. "Módulo de capacitación para la recolección y el análisis de indicadores de innovación”. Banco Interamericano de Desarrollo 8 (1): 10-12. https://bit. ly/37IbQsP.

Mendizábal, Guillermo. 2002. "Estrategias para la innovación tecnológica en Castillas y León”. Tesis doctoral, Universidad de Valladolid, Valladolid. https://bit.ly/3fQVTVv.

Organización Mundial de la Propiedad Intelectual (OMPI). 2019. Global Innovation Index: Creating Healthy Lives-The Future. Accedido abril de 2019. https://bit.ly/3mJnCaH.

---. 2020. Global Innovation Index: Who Will Finance Innovation? Accedido mayo de 2019. https://bit.ly/3fSg8Co.

Organización para la Cooperación y el Desarrollo Económicos (OCDE) y Oficina de Estadística de las Comunidades Europeas (Eurostat). 2005. "Manual de Oslo: guía para la recogida e interpretación de datos sobre innovación”. París: OCDE. https://doi.org/10.1787/9789264013100-en.

---. 2018. Manual de Oslo: directrices para recopilar, informar y utilizar datos sobre innovación, cuarta edición. Paris: OCDE. https://doi.org/10.1787/9789264304604-en. 
Ortega, Juan. 2019. "Determinantes del esfuerzo en innovación en empresas seguidoras en economías en desarrollo: el caso de la industria manufacturera de Colombia". Tesis doctoral, Universidad Politécnica de Valencia, Valencia. https://bit.ly/3g4roMb.

Padilla, Ramón. 2006. "Conceptos de competitividad e instrumentos para medirla". Revista CEPAL 5 (1): 23-56. https://bit.ly/3piltEi.

Pech, José, y María Morales. 2000. "Competitividad y estrategia: el enfoque de competencias esenciales y el enfoque basado en los recursos". Revista Contaduría y Administración 10 (197): 34-56. https://bit.ly/3mPpKha.

Pita, Salvador, y Sonia Pértegas. 2002. "Investigación cuantitativa y cualitativa". Cad Aten Primaria 9 (1): 76-78. https://bit.ly/3gLGF4G.

Porter, Michael. 1990. "The Competitive Advantage of Nations". Harvard Business Review 1 (6): 64-87. https://doi.org/10.1007/978-1-349-11336-1_5.

Reichstein, Toke, y Ammon Salter. 2006. "Investigar las fuentes de innovación de procesos entre las empresas manufactureras del Reino Unido". Cambio Industrial y Corporativo 15 (4): 653-682. https://doi.org/10.1093/icc/dt1014.

Roberts, Peter, y Raphael Amit. 2003. "The Dynamics of Innovative Activity and Competitive Advantage: The Case of Australian Retail Banking 1981 to 1995". Organization Science 14 (2): 107-122. https://doi.org/10.1287/orsc.14.2.107.14990.

Romero María, Alfredo Rébori y María Camino. 2010. "Un índice para medir el nivel de innovación tecnológica en empresas intensivas en el uso de tecnología". Revista de Administração e Inovação 7 (1): 3-20. https://doi.org/10.5585/rai.v7i1.578.

Saavedra, María. 2017. "Una propuesta para la determinación de la competitividad en la pyme latinoamericana". Pensamiento \& Gestión 5 (33): 2-32. https://bit.ly/3nIsoXf.

Sánchez Yesenia, Francisco García y José Mendoza. 2015. "La capacidad de innovación y su relación con el emprendimiento en las regiones de México". Estudios Gerenciales 31 (136): 243-252. https://doi.org/10.1016/j.estger.2015.04.001.

Santillán, José. 2010. "Competitividad de las micro y pequeñas empresas constructoras dedicadas a la Edificación en el Distrito Federal". Tesis de maestría, Universidad Nacional Autónoma de México, Ciudad de México. https://bit.ly/3aBLdYj.

Schnarch, Alejandro. 2007. "Creatividad, innovación y entrepreneurship". Revista Recrearte 2 (7): 121-134. https://bit.ly/3aApKio.

Schumpeter, Joseph. 1934. The Theory of Economic Development. Cambridge: Harvard University. http://bit.ly/2LggRjz.

Solleiro, José, y Rosario Castañón. 2015. "Competitividad y sistemas de innovación: los retos para la inserción de México en el contexo global”. Temas de Iberoamérica 6 (11): 1-34. https://doi.org/10.1016/j.technovation.2004.02.005.

Superintendencia de Economía Popular y Solidaria (SEPS). 2018. Ley Orgánica de Economía Popular y Solidaria. Registro Oficial 444, 10 mayo. https://bit.ly/31T2oyP.

---. 2019. Catastro sector no financiero. Accedido enero de 2019. http://bit.ly/2X4U0tQ. 
Torres Natali, Pablo López y Alodia Alemán. 2017. "Balance de la Economía Popular y Solidaria en Ecuador". Revista Economía y Desarrollo 24 (85): 12-34. https://bit.ly/3o9W4wy.

Vélez, Cecilia, Sergio Afcha y Miguel Bustamante. 2019. "Cooperación universidad-empresa y su efecto sobre el desempeño innovador". Información Tecnológica 30 (1): 4-20. http:// dx.doi.org/10.4067/S0718-07642019000100159.

Yam, Richard, William Lo, Esther Tang y Antonio Lau. 2004. "Analysis of Sources of Innovation, Technological Innovation Capabilities, and Performance: An Empirical Study of Hong Kong Manufacturing Industries". Research Policy 40 (3): 391-402. https://doi. org/10.1016/j.respol.2010.10.013.

Zayas, Imelda, Daniela Parra, Rosa López y Juan Torres. 2015. "La innovación, competitividad y desarrollo tecnológico en las mipyme del municipio de Angostura, Sinaloa". Revista Mexicana de Ciencias Agrícolas 6 (3): 30-50. https://doi.org/10.29312/remexca.v6i3.642.

Zoltán, Juan, Szerb László y Lloyd Ainsley. 2018. Global Entrepreneurship Index. Washington D. C.: GEDI. https://bit.ly/2R4SBng. 